\title{
Drug therapies in older adults (part 1)
}

\author{
Authors: Omar Mukhtar ${ }^{A}$ and Stephen HD Jackson ${ }^{B}$
}

Prescribing for older adults represents a significant challenge as the UK population ages. Physiological decline and the rising prevalence of frailty increase the likelihood of altered pharmacodynamics and pharmacokinetics, suboptimal prescribing and adverse effects among this growing cohort of the population. In the first of two articles, we begin by considering these issues and posit four key questions which should be considered when prescribing for older adults. Does this agent reflect the priorities of the patient? Are there alternatives - with greater efficacy, effectiveness or tolerability - that might be considered? Are the dose, frequency and formulation appropriate? How does this prescription relate to concurrent medication? We also describe current drug therapies in two disease states with a predilection for older adults: Alzheimer's disease (AD) and osteoporosis. Using these examples we highlight the limitations of evidencebased medicine and guidelines in this cohort of the population, illustrating the reliance on sub-group analysis to demonstrate the efficacy of drug therapies for older adults in osteoporosis and the underutilisation of appropriate treatments for patients with $A D$ as a result of flawed guidelines.

KEYWORDS: Pharmacology, elderly, geriatrics, physiology of ageing, prescribing, osteoporosis, Alzheimer's disease, guidelines

\section{Introduction}

The UK's population is ageing. The percentage of people aged 65 years and over increased from 15\% in 1985 to $17 \%$ in 2010 - an absolute increase of 1.7 million people. By 2035 it is predicted that this cohort will account for $23 \%$ of the total population. ${ }^{1}$ The fastest increase is likely to occur among the 'oldest old' (those aged 85 years and over) - between 1985 and 2010 , this population more than doubled from approximately 0.7 million to greater than 1.4 million people. ${ }^{1}$

In total, $45 \%$ of the medications prescribed in the UK are for individuals aged over 65 years, and $36 \%$ of people aged 75 years and above take four or more prescribed drugs on a daily basis. ${ }^{2}$ However, with increased variability in the risks

Authors: ASpR in clinical pharmacology and therapeutics, King's Health Partners, King's College Hospital, London, UK; ' Bprofessor of clinical gerontology, Department of Clinical Gerontology, King's Health Partners, King's College Hospital, London, UK and benefits of drugs, along with a relatively limited evidence base, prescribing for this population remains problematic. ${ }^{3-5}$ This first review highlights a number of key issues relevant to those who prescribe for older adults and describes current drug therapies in two examples of highly age-associated disease states: Alzheimer's disease (AD) and osteoporosis.

\section{Prescribing considerations in older adults}

\section{Ageing and frailty}

Physiological ageing is best described as the time-related loss of functional units within an organ system, eg nephrons in the kidneys, alveoli in the lungs and neurones in the brain. By contrast, frailty is defined by progressive physiological decline in multiple organ systems resulting in loss of function, loss of physiological reserve and increased vulnerability to disease and death. ${ }^{6}$ In recent years, the recognition of this distinction has led to an awareness that frail adults, rather than older people per se, are the primary users of healthcare resources. It can, therefore, be difficult to give useful prescribing advice on the basis of age alone - a housebound 63 year old may be frailer than a 78 year old who continues to work on a part-time basis. ${ }^{6}$

\section{Pharmacokinetic and pharmacodynamic changes}

With physiological ageing, the body undergoes several changes that can affect the pharmacokinetics of drugs (Table 1). ${ }^{7}$ The most significant of these is the reduction in renal clearance, which results in the reduced excretion of water-soluble agents variations in drug distribution and metabolism are also noteworthy (Table 1).

Furthermore, pharmacodynamic changes increase the sensitivity of older adults to prescribed therapy. This is particularly true for drugs which act via the central nervous system: neuroleptic use in older adults is associated with increased rates of delirium, extrapyramidal symptoms and postural hypotension, while the benzodiazepines are associated with increased body sway and exaggerated sedative effects. Equally, agents with anticholinergic actions impair cognition and orientation, particularly in patients with a pre-existing cholinergic deficit eg AD.?

\section{Polypharmacy and appropriate prescribing}

The use of many medicines ('polypharmacy') is common among older people; a recent primary care survey in Scotland found that $28.6 \%$ of adults aged $60-69$ years were receiving 
Table 1. Major physiological changes observed with ageing and their pharmacokinetic effects.

\begin{tabular}{|c|c|c|c|}
\hline $\begin{array}{l}\text { Pharmacokinetic } \\
\text { process }\end{array}$ & Physiological change & Pharmacokinetic effect & Drugs most affected \\
\hline \multirow[t]{2}{*}{ Distribution } & \multirow{2}{*}{$\begin{array}{l}\text { Decreased total body mass, increased } \\
\text { proportion of body fat and decreased } \\
\text { proportion of body water }\end{array}$} & $\begin{array}{l}\text { Increased } V_{d} \text { of highly lipid soluble } \\
\text { drugs }\end{array}$ & $\begin{array}{l}\text { Benzodiazepines, morphine and } \\
\text { amiodarone }\end{array}$ \\
\hline & & Decreased $V_{d}$ of hydrophilic drugs & $\begin{array}{l}\text { Gentamicin, digoxin, lithium and } \\
\text { theophylline }\end{array}$ \\
\hline Metabolism & $\begin{array}{l}\text { Reduced hepatic mass, hepatic blood } \\
\text { flow and metabolic capacity }\end{array}$ & $\begin{array}{l}\text { Drugs with a high } \mathrm{E} \text { are associated } \\
\text { with the largest reductions in hepatic } \\
\text { clearance }\end{array}$ & $\begin{array}{l}\text { Clomethiazole, dextropropoxyphene, } \\
\text { glyceryl trinitrate, lidocaine, } \\
\text { pethidine and propranolol }\end{array}$ \\
\hline Excretion & $\begin{array}{l}\text { Reduced glomerular filtration rate, renal } \\
\text { tubular function and renal blood flow }\end{array}$ & $\begin{array}{l}\text { Accumulation of renally cleared } \\
\text { drugs }\end{array}$ & $\begin{array}{l}\text { Digoxin, lithium, gentamicin and } \\
\text { ACEi }\end{array}$ \\
\hline
\end{tabular}

between four and nine medications, while $7.4 \%$ were receiving ten or more; among those aged 80 years and over, the figures were 51.8 and $18.6 \%$, respectively. ${ }^{8}$ While the published literature suggests that such polypharmacy is associated with adverse clinical outcomes and increased healthcare costs, such analyses fail to account for the appropriateness (or otherwise) of the prescribed drugs. ${ }^{9,10}$

The term appropriate prescribing has far greater clinical relevance; it addresses both the problem of inappropriate medication use and the failure to prescribe or use appropriate treatments. This latter aspect of appropriate prescribing is frequently neglected (particularly among older adults). With regard to the former, patients taking more than five medications are approximately three times more likely to receive an inappropriately prescribed drug. ${ }^{11}$

\section{The evidence base}

In order to determine whether a treatment is appropriate, it is necessary to have an idea of its relative risks and benefits. Clinicians routinely base this assessment on their interpretation of clinical trials. While these provide evidence of the relative benefits of a treatment, they are rarely powered to determine differences in uncommon or rare adverse effects - sequelae which often have significant clinical consequences for individual patients. ${ }^{12}$ Moreover, the application of trial data to older adults is problematic as subjects over the age of 65 or 75 years are frequently excluded, despite the fact that many patients with the disease process being studied are in their eighties or nineties. ${ }^{6}$ The process of extrapolation should, therefore, be applied with caution. Although there is some evidence that the efficacy of drug treatments does not differ significantly between older adults and adults in general, important discrepancies may occur. For example, in metastatic breast cancer, tumour response rates to chemotherapy (intervention) versus hormone therapy (control) are age dependent: in older adults ( $\geq 60$ years) the odds ratio (OR) of a response is 1.60 with a $95 \%$ confidence interval (CI) of $0.95-2.72$ versus an OR of 0.51 with a $95 \%$ CI of $0.26-0.99$ in younger adults ( $<60$ years).$^{13}$

\section{Practical prescribing for the older adult}

Good prescribing practices may limit adverse effects and inappropriate prescribing among older adults. While some of these practices are evidence based, most are consensus statements, due to limited study data. Electronic prescribing, educational initiatives and a limitation in the number of prescribers demonstrably improve the quality of prescribing; ${ }^{14-16}$ however, medication reviews and new prescription checklists may offer the greatest potential to improve prescribing for older adults.

The 'National Service Framework for Older People', published over a decade ago, recommended regular medication reviews: for patients receiving four or more drugs a review was suggested at six-monthly intervals, while an annual review was suggested for those taking three or fewer medicines. ${ }^{17}$ For each prescription, we propose that prescribers consider four key questions.

1. Does this agent reflect the priorities of the patient?

2. Are there alternatives - with greater efficacy, effectiveness or tolerability - that might be considered?

3. Are the dose, frequency and formulation appropriate?

4. How does this prescription relate to the concurrent medication?

While more formal approaches have sought to list medications that may be inappropriate for older adults, eg the NSF prescribing indicators ${ }^{17}$ or STOPP/START checklists, ${ }^{18}$ the application of these is challenging in routine clinical practice. As an alternative, the four questions listed above should lead to the cessation of inappropriate therapies and the judicious use of new drugs.

\section{Alzheimer's Disease}

$\mathrm{AD}$ is the commonest cause of dementia worldwide, with its global prevalence estimated to be as high as 24 million people. This figure is set to double every 20 years until the middle of the 21 st century. ${ }^{19} \mathrm{AD}$ is a chronic neurodegenerative condition characterised by progressive cognitive deficits which are thought to correlate with neuronal loss, particularly in the hippocampus and cerebral neocortex. ${ }^{20}$ Although extracellular $\beta$-amyloid plaques and intraneuronal neurofibrillary tangles (composed of tau protein) are a feature of physiological ageing, the burden of these lesions, particularly amyloid plaques, is far greater in individuals who develop AD. ${ }^{21}$ 
Various pharmacological approaches have been pursued in an attempt to modify disease progression. Most have been unsuccessful - in 2013, Eli Lilly reported the failure of Semagacestat, an inhibitor of $\gamma$-secretase (an enzyme integral to the production of $\beta$-amyloid). ${ }^{22}$ In 2012, the development of bapineuzumab, a humanised monoclonal antibody which targets $\beta$-amyloid, was also discontinued after it failed to demonstrate efficacy in two phase III trials. ${ }^{23}$ Those targeting tau formation have experienced similar disappointment: AL-108 (Allon Therapeutics Inc), an intranasal formulation of davunetide (an eight amino-acid peptide thought to decrease tau phosphorylation and maintain the attachment of tau protein to the microtubular network) failed to demonstrate efficacy in a phase II/III clinical trial. ${ }^{24}$ Equally, the kinase inhibitor, tideglusib, which aimed to inhibit the phosphorylation of tau proteins, failed to demonstrate clinical efficacy in $\mathrm{AD}^{25}$

As a result, the cholinesterase inhibitors donepezil, galantamine and rivastigmine remain the mainstays of treatment for the relief of mild/moderate $\mathrm{AD}$, while memantine is licenced for the treatment of cognitive deficits arising from moderate/severe Alzheimer's dementia. Potential treatments for the neuropsychiatric symptoms observed in $\mathrm{AD}$ are also limited, with risperidone the only drug licensed in the UK for the treatment of behavioural and psychological symptoms arising from dementia.

\section{Cholinesterase inhibitors}

The use of cholinesterase inhibitors for patients with mild/ moderate cognitive impairment in $\mathrm{AD}$ is predicated upon the cholinergic hypothesis. ${ }^{26}$ This implies that cholinergic deficits are responsible for the cognitive and behavioural changes observed. Accordingly, three therapeutic interventions have been trialled: the use of acetylcholine precursors, cholinergic agonists and cholinesterase inhibitors. ${ }^{27}$ The former approaches, using several different drugs, have consistently failed to demonstrate clinically significant efficacy. ${ }^{27}$ By contrast, the cholinesterase inhibitors appear to slow the rate of cognitive, functional and behavioural decline in patients with $\mathrm{AD} .{ }^{28}$ Of the four commercially produced compounds, only three are currently available in the UK and approved by the National Institute for Health and Care Excellence (NICE). ${ }^{29}$ Tacrine, the first licensed drug, is no longer actively marketed in view of its hepatotoxicity. ${ }^{27}$

\section{Donepezil}

Donepezil is a centrally acting reversible acetylcholinesterase inhibitor: with an elimination half-life of approximately 70 hours, it can be taken once a day. ${ }^{27}$ It has been the primary focus of over 200 trials, the vast majority of which focus on its role in AD. A 2006 Cochrane review of all double-blind, randomised, placebo-controlled trials of donepezil for patients with mild, moderate or severe Alzheimer's dementia, found benefits in cognitive function, activities of daily living and behaviour among all three groups. ${ }^{30}$ Benefits arising from a dose of $10 \mathrm{mg}$ per day were significantly greater than those seen with $5 \mathrm{mg}$ per day. ${ }^{30}$ However, the 2006 NICE guidance recommended that donepezil only be used for patients with mild $\mathrm{AD} ;{ }^{31}$ the 2011 guidance extended this indication to moderate $\mathrm{AD} .^{29}$ Despite this, data from subsequent trials continues to demonstrate the efficacy of donepezil in moderatesevere $\mathrm{AD}$, eg the DOMINO trial. ${ }^{32}$

\section{Galantamine}

Galantamine is a competitive, reversible inhibitor of acetylcholinesterase. ${ }^{27}$ It also functions as an allosteric modulator of nicotinic receptors, which may lead to the increased release of acetylcholine. ${ }^{33}$ Its efficacy has been demonstrated at doses of 8 and $16 \mathrm{mg}$ twice a day, with fewer adverse effects observed at the lower dose. ${ }^{27}$ Inhibitors of CYP2D6 or 3A4 (eg paroxetine, amitriptyline, fluoxetine, quinidine and ketoconazole) may decrease the clearance of galantamine, thereby increasing the potential for central (eg excitation or agitation) or peripheral (eg bradycardia or digestive disorders) hypercholinergic effects. ${ }^{27}$

Until recently, the safety and efficacy of galantamine had only been documented in randomised controlled trials of $<6$ months duration. ${ }^{34,35}$ However, in 2013, results from a pan-European, two-year, randomised, double-blind, placebo-controlled trial of galantamine in mild/moderately severe $\mathrm{AD}$ were reported (mean age: 73 years). Its primary outcome measures were allcause mortality and change in mini-mental state examination (MMSE) score (baseline vs 24 months). ${ }^{36}$ Despite a withdrawal rate of $27 \%$, the trial was terminated early, not on the basis of MMSE scores (placebo - mean MMSE: -2.14 (standard deviation (SD) 4.34); galantamine - mean MMSE: 1.41 (SD $4.05)$; $\mathrm{p}<0.001$ ) but due to a significantly lower mortality rate among those receiving active treatment (hazard ratio (HR): 0.58; 95\% CI: 0.37-0.89; $\mathrm{p}=0.011) .{ }^{36}$

\section{Rivastigmine}

In contrast to donepezil and galantamine, rivastigmine is a pseudo-irreversible cholinesterase inhibitor which acts on both acetylcholinesterase and butyrylcholinesterase. ${ }^{27}$ It is currently licensed in the UK for the treatment of mild/ moderate dementia secondary to Alzheimer's and Parkinson's disease. ${ }^{37,38}$ In both instances, it has demonstrated efficacy, with a 2009 Cochrane review reporting delayed cognitive decline, improvements in activities of daily living and favourable behavioural patterns among adults with mild/ moderate $\mathrm{AD}$ receiving rivastigmine, when compared with placebo. $^{39}$

However, oral rivastigmine is associated with significant rates of nausea and vomiting, which may be a feature of its dual inhibitory mechanism. ${ }^{40}$ As a result, two transdermal patches have been formulated (17.4 and $9.5 \mathrm{mg}$ ) with equivalent efficacy, but fewer adverse effects, when compared with capsules. $^{41}$

\section{Memantine}

Neuronal death is associated with the overstimulation of $N$-methyl-D-aspartate (NMDA) receptors and excessive glutamate exposure, leading to an increase in intracellular calcium. ${ }^{42}$ Memantine, an NMDA receptor antagonist, is thought to block this ion channel, preventing the influx of calcium and the excitotoxicity of neurones. ${ }^{42}$

While preclinical studies and animal models support the concurrent use of both memantine and the cholinesterase inhibitors in moderate/severe $\mathrm{AD}$, multiple meta-analyses and 
systematic reviews have failed to show a consistent benefit for this approach in humans. ${ }^{29,43,44}$ Equally, the trial data does not demonstrate consistent efficacy for the use of memantine in patients with mild AD. ${ }^{45}$ Thus, despite a pharmacologically plausible mechanism of action, memantine is currently only licensed for the treatment of moderate/severe AD $(\text { MMSE }<20)^{29}$

\section{Osteoporosis}

Osteoporosis is predominantly a disease of older adults in which compromised bone density and quality predispose both men and women to an increased risk of fractures. Underdiagnosis remains an issue, particularly among men. ${ }^{46}$ While the incidence of hip fractures increases exponentially with age, this increase is more pronounced than might be expected from the age-related decline in bone mass alone. ${ }^{47-49}$ In keeping with this, a number of risk factors for osteoporosis have been identified, including a low body mass index, family history, smoking, excessive alcohol intake and long-term glucocorticoid use. ${ }^{4-49}$

In 2008, the Fracture Risk Assessment Tool (FRAX) was developed, which sought to incorporate these clinical risk factors, among others, into the assessment of a patient's fracture risk with or without prior knowledge of bone mineral density (BMD) at the hip. ${ }^{49}$ Specifically, it establishes the 10 -year probability of any major osteoporotic fracture (clinically relevant spine, forearm, hip or shoulder fracture) while delineating the 10-year risk of hip fracture in isolation. ${ }^{49}$

Based on current evidence, the consensus opinion for the prevention of osteoporosis is to recommend avoidance of smoking, excess alcohol and caffeine $;^{50}$ fall prevention measures should also be adopted along with structured exercise ${ }^{50}$ Calcium (1,000-1,200 mg) should be consumed on a daily basis (either via diet or supplementation) along with 800-1,000 U vitamin D; however, the evidence for this specific measure in osteoporosis has recently been called into question. ${ }^{50,51}$ The treatment of postmenopausal osteoporosis should include the correction of any calcium deficiency, continuation of preventative measures and additional pharmacological therapy. ${ }^{50}$ Drugs that have been licenced in addition to preventative oestrogen therapy (for women) include the bisphosphonates, raloxifene, parathyroid hormone, denosumab and calcitonin.

\section{Bisphosphonates}

Bisphosphonates reduce the local recruitment of osteoclasts, impair their resorptive function and accelerate their demise. ${ }^{46}$ In doing so, they prevent bone loss and increase BMD. ${ }^{46} \mathrm{Of}$ the five agents currently licenced in the UK for the treatment of osteoporosis - disodium etidronate, alendronic acid, risedronate sodium, ibandronic acid and zoledronic acid the weekly oral preparation, alendronate (alendronic acid), represents the first line of treatment, owing to its efficacy, longterm safety data and favourable cost.

A 2008 Cochrane review assessed the efficacy of alendronate in the prevention of osteoporotic fractures among postmenopausal women. ${ }^{52}$ Across 11 trials, with some subjects aged over 90 years, alendronate use was associated with a significant reduction in the absolute risk of vertebral
(6\%), non-vertebral (2\%), hip (1\%) and wrist (2\%) fractures among women with a $\mathrm{T}$ score of $\leq-2$ and/or $\geq 1$ vertebral compression fracture. ${ }^{52}$ Retrospective analysis of the Fracture Intervention Trial indicates that the relative risk reductions (RRRs) achieved are likely to be similar across all age groups. In this analysis, the greatest absolute benefit was observed among those aged over 75 years, due to the incident rate of fractures. ${ }^{53}$ Despite this efficacy data, adherence to oral bisphosphonates remains poor, with 12-month adherence estimated to be approximately $60 \% .^{54}$

Although osteonecrosis of the jaw is a well publicised adverse effect associated with the bisphosphonates, it is rare (incidence rate: $<1$ in 10,000/patient-treatment years), particularly within the context of those receiving oral bisphosphonates for osteoporosis. ${ }^{55}$ Good oral hygiene, regular dental checkups and heightened care when undergoing periodontal procedures may help prevent this complication. ${ }^{55}$ Similarly, there is a small risk that bisphosphonate therapy may be associated with atypical subtrochanteric fractures (incidence rate: $32 /$ million patient-years). ${ }^{56}$ This risk may be exaggerated among those receiving treatment for $>5$ years, ${ }^{56}$ and patients who experience such a fracture may benefit from teriparatide treatment as an alternative.$^{57}$ Controversy regarding the bisphosphonates also relates to a possible association with oesophageal cancer. Predicated upon a series of case reports, the Medicines and Healthcare Products Regulatory Agency sought to evaluate this issue by means of an epidemiological study. ${ }^{58}$ The results suggested that five years or more of bisphosphonate therapy was associated with a small absolute increase in the risk of oesophageal cancer among men and women aged between $60-79$ years. ${ }^{58}$ While others have not observed this increased risk, the spectre of this association has yet to be definitively excluded. ${ }^{59,60}$

\section{Denosumab}

Denosumab is a humanised monoclonal antibody to the receptor activator of the nuclear factor, kappa-B ligand (RANKL), an osteoclast-differentiating factor. By preventing the binding of RANKL to RANK on osteoclast precursors and osteoclasts, it reduces bone resorption.

However, a formal evaluation of denosumab in older adults has yet to take place. Subgroup analysis of subjects aged 75 years or older in the Fracture Reduction Evaluation of Denosumab in Osteoporosis Every 6 Months (FREEDOM) trial (subcutaneous administration of denosumab - $60 \mathrm{mg}$ every six months) revealed that 3 years of treatment significantly reduced the risk of hip fractures $(2.3 \%$ placebo vs $0.9 \%$ denosumab; $\mathrm{p}<0.01)$; the risk reduction was similar to that observed among those under the age of 75 years. ${ }^{61}$ In the associated extension trial (mean age: 75 years) denosumab was associated with a progressive increase in BMD and a sustained, but non-progressive decrease in bone turnover; while yearly fracture rates were below those observed among subjects in receipt of placebo during the FREEDOM trial, they were greater than those in receipt of active treatment. ${ }^{62}$ Infections, eczema, musculoskeletal pain and hypercholesterolemia have all been observed as adverse effects and there is a suspicion that denosumab may also lead to osteonecrosis of the jaw, atypical fractures and delayed fracture healing, at rates comparable to the bisphosphonates. ${ }^{63}$ 


\section{Raloxifene}

Like tamoxifen, raloxifene is a selective oestrogen receptor modulator), a compound which binds to oestrogen receptors and modulates (through agonism and antagonism) the effects of oestrogen in a variety of tissues. Approved for the prevention and treatment of osteoporosis in postmenopausal women, raloxifene also reduces the risk of invasive breast cancer. ${ }^{64}$ In the Multiple Outcomes of Raloxifene Evaluation (MORE) trial (mean age: 69 years) the risk of vertebral fracture was reduced among subjects receiving raloxifene (30\% RRR: $60 \mathrm{mg} /$ day; 50\% RRR: $120 \mathrm{mg} /$ day) while the reduction in non-vertebral fractures did not reach significance; there were no differences in safety or effectiveness between those aged $>75$ years and their younger counterparts. ${ }^{65}$ However, with an increased risk of deep vein thrombosis, pulmonary embolism and fatal stroke, raloxifene is contraindicated in patients with a prior history of venous thromboembolic disease and caution should be exercised when prescribing for those with known cardiovascular disease. ${ }^{65}$ Equally, adverse reactions relating to its hormonal mechanism limit its use among men and older women.

\section{Calcitonin}

Salmon calcitonin - available as both a subcutaneous injection and nasal spray - acts directly on osteoclasts to inhibit bone resorption. The Prevent Recurrence of Osteoporotic Fractures (PROOF) study in postmenopausal women with osteoporosis (mean age: 69 years) demonstrated that 200 IU salmon calcitonin per day, delivered via a nasal spray, decreased the absolute risk of new vertebral fractures by $8 \%$ at the end of 5 years. ${ }^{66}$ However, the reduction in non-vertebral fractures did not reach significance and the discontinuation rate at the end of the study was 59\%. ${ }^{67}$ While calcitonin use is associated with a risk of serious allergic reactions, hypocalcaemic tetany, nausea, diarrhoea, flushing and nasal irritation, it is no longer recommended for the prevention or treatment of osteoporosis due to its association with malignancy. ${ }^{67}$

\section{Transdermal parathyroid hormone}

In contrast to the other agents described, teriparatide (parathyroid hormone) is an anabolic treatment which preferentially increases bone formation. Again, its use in women aged 75 years and older is dependent upon subgroup analysis, as opposed to a double-blind, randomised controlled trial designed to evaluate its efficacy specifically in older adults. Such an analysis of the Fracture Prevention Trial (FPT) suggests that teriparatide treatment may be associated with a significant reduction in the risk of new vertebral fractures (RRR 65\%; p < 0.05) in older women (aged $\geq 75$ years); however, the reduction in non-vertebral fractures did not reach significance. ${ }^{68}$ Originally administered as a daily subcutaneous injection, a transdermal patch is now available, which is effective in increasing BMD at the lumbar spine and hip in postmenopausal women with osteoporosis over a period of six months. ${ }^{69}$

However, no fracture data is available with this new preparation and the risk of transient hypocalcaemia is significantly increased in those receiving teriparatide (24\% of patients vs $3 \%$ in the placebo group). This, coupled with an increased incidence of osteosarcoma in animal studies, has led to a recommendation that treatment be limited to $<2$ years, and only administered to patients who have 'failed' bisphosphonate therapy. ${ }^{69,70}$

\section{Concluding thoughts}

Prescribing for older adults represents a significant challenge. Physiological decline, frailty and multiple pathologies increase the likelihood of altered pharmacodynamics and pharmacokinetics, suboptimal prescribing and adverse effects among this growing cohort of the population. The persistent failure of evidence-based medicine to represent these patients adds to this challenge. While many of the landmark trials in osteoporosis illustrate the reliance on sub-group analysis to demonstrate the efficacy of drug therapies for older adults, the current guidelines for the pharmacological management of $\mathrm{AD}$ reinforce the issue of underutilisation of appropriate treatments, for this oft-neglected group.

\section{References}

1 Office for National Statistics. Population ageing in the United Kingdom, its constituent countries and the European Union. London: HMSO, 2012.

2 Wynne HA, Blagburn J. Drug treatment in an ageing population: practical implications. Maturitas 2010;66:246-50.

3 Lipsitz L. Minireview: Altered blood pressure homeostasis in advanced age clinical and research implications. J Geront 1989;44:179-83.

4 Masoudi FA, Havranek EP, Wolfe P et al. Most hospitalized older persons do not meet the enrollment criteria for clinical trials in heart failure. Am Heart J 2003;146:250-7.

5 McMurdo MET, Roberts H, Parker S et al; Age and Ageing Specialty Group, NIHR, Comprehensive Clinical Research Network. Improving recruitment of older people to research through good practice. Age Ageing 2011;40:659-65.

6 Walston J, Hadley EC, Ferrucci L et al. Research agenda for frailty in older adults: toward a better understanding of physiology and etiology: summary from the American Geriatrics Society/National Institute on Aging Research Conference on Frailty in Older Adults. J Am Geriatr Soc 2006;54:991-1001.

7 Mangoni AA, Jackson SHD. Age-related changes in pharmacokinetics and pharmacodynamics: basic principles and practical applications. Br J Clin Pharmacol 2004;57:6-14.

8 Payne RA, Avery AJ, Duerden M et al. Prevalence of polypharmacy in a Scottish primary care population. Eur J Clin Pharmacol 2014;70:575-81.

9 Campbell SE, Seymour DG, Primrose WR. A systematic literature review of factors affecting outcome in older medical patients admitted to hospital. Age Ageing 2004;33:110-5.

10 Frazier SC. Health outcomes and polypharmacy in elderly individuals. J Gerontol Nursing 2005;31:4-11.

11 Steinman MA, Landefeld CS, Rosenthal GE et al. Polypharmacy and prescribing quality in older people. J Am Geriatr Soc 2006;54:1516-23.

12 Loke YK, Golder S, Vandenbroucke JP. Comprehensive evaluations of the adverse effects of drugs: importance of appropriate study selection and data sources. Therap Adv Drug Safety 2011;2:59-68.

13 Seegers V, Trinquart L, Boutron I et al. Comparison of treatment effect estimates for pharmacological randomized controlled trials enrolling older adults only and those including adults: a metaepidemiological study. PLoS One 2013;8:e63677.

14 Green JL, Hawley JN, Rask KJ. Is the number of prescribing physicians an independent risk factor for adverse drug events in an elderly outpatient population? Am J Geriatr Pharmacother 2007;5:31-9. 
15 Franklin BD, O'Grady K, Donyai P et al. The impact of a closed-loop electronic prescribing and automated dispensing system on the ward pharmacist's time and activities. Int J Pharmacy Pract 2007;15:133-9.

16 O'Brien T, Oxman AD, Davis DA et al. Educational outreach visits: effects on professional practice and health care outcomes. Cochrane Database Syst Rev 2000;2:CD000409.

17 Department of Health. National service framework for older people. London: HMSO, 2001

18 Gallagher P, Ryan C, Byrne S et al. STOPP (Screening Tool of Older Person's Prescriptions) and START (Screening Tool to Alert doctors to Right Treatment). Consensus validation. Int J Clin Pharmacol Ther 2008;46:72-83.

19 Reitz C, Brayne C, Mayeux R. Epidemiology of Alzheimer disease. Nat Rev Neurol 2011;7:137-52.

20 Brun A, Englund E. Regional pattern of degeneration in Alzheimer's disease: neuronal loss and histopathological grading. Histopathology 1981;5:549-64.

21 Dubois B, Feldman HH, Jacova C et al. Revising the definition of Alzheimer's disease: a new lexicon. Lancet Neurol 2010;9:1118-27.

22 Doody RS, Raman R, Farlow M et al. A phase 3 trial of semagacestat for treatment of Alzheimer's disease. N Engl J Med 2013;369:341-50.

23 Salloway S, Sperling R, Fox NC et al. Two phase 3 trials of bapineuzumab in mild-to-moderate Alzheimer's disease. $N$ Engl J Med 2014;370:322-33.

24 Morimoto BH, Schmechel D, Hirman J et al. A double-blind, placebocontrolled, ascending-dose, randomized study to evaluate the safety, tolerability and effects on cognition of AL-108 after 12 weeks of intranasal administration in subjects with mild cognitive impairment. Dement Geriatr Cogn Disord 2013;35:325-36.

25 del Ser T, Steinwachs KC, Gertz HJ et al. Treatment of Alzheimer's disease with the GSK-3 inhibitor tideglusib: a pilot study. J Alzheimers Dis 2013;33:205-15.

26 Bartus R, Dean R, Beer B et al. The cholinergic hypothesis of geriatric memory dysfunction. Science 1982;217:408-14.

27 Schneider LS. Alzheimer disease pharmacologic treatment and treatment research. Continuum 2013;19:339-57.

28 Birks J. Cholinesterase inhibitors for Alzheimer's disease. Cochrane Database Syst Rev 2009;1:CD005593.

29 National Institute for Health and Care Excellence. Donepezil, galantamine, rivastigmine and memantine for the treatment of Alzheimer's disease. TA217. London: NICE, 2011.

30 Birks J, Harvey RJ. Donepezil for dementia due to Alzheimer's disease. Cochrane Database Syst Rev 2006;1:CD001190.

31 National Institute for Health and Care Excellence. Dementia: Supporting people with dementia and their carers in health and social care. CG42. London: NICE, 2006.

32 Howard R, McShane R, Lindesay J et al. Donepezil and memantine for moderate-to-severe Alzheimer's disease. N Engl J Med 2012;366:893-903.

33 Woodruff-Pak DS, Vogel RW 3rd, Wenk GL. Galantamine: Effect on nicotinic receptor binding, acetylcholinesterase inhibition, and learning. Proc Natl Acad Sci USA 2001;98:2089-94.

34 Raskind MA, Peskind ER, Wessel T et al. Galantamine in AD: A 6-month randomized, placebo-controlled trial with a 6-month extension. The Galantamine USA-1 Study Group. Neurol 2000;54:2261-8.

35 Wilcock GK, Lilienfeld S, Gaens E. Efficacy and safety of galantamine in patients with mild to moderate Alzheimer's disease: multicentre randomised controlled trial. Galantamine International-1 Study Group. BMJ 2000;321:1445-9.

36 Hager K, Baseman AS, Nye JS et al. Effects of galantamine in a 2-year, randomized, placebo-controlled study in Alzheimer's disease. Neuropsychiatr Dis Treat 2014;10:391-401.

37 Touchon J, Bergman H, Bullock R et al. Response to rivastigmine or donepezil in Alzheimer's patients with symptoms suggestive of concomitant Lewy body pathology. Curr Med Res Opin 2006;22:49-59.
38 Burn D, Emre M, McKeith I et al. Effects of rivastigmine in patients with and without visual hallucinations in dementia associated with Parkinson's disease. Mov Disord 2006;21:1899-907.

39 Birks J, Grimley Evans J, Iakovidou V, Tsolaki M, Holt FE. Rivastigmine for Alzheimer's disease. Cochrane Database Syst Rev 2009;2:CD001191.

40 Inglis F. The tolerability and safety of cholinesterase inhibitors in the treatment of dementia. Int J Clin Pract Suppl 2002;127:45-63.

41 Cummings J, Lefevre G, Small G et al. Pharmacokinetic rationale for the rivastigmine patch. Neurol 2007;69:S10-3.

42 Danysz W, Parsons CG, Mobius HJ et al. Neuroprotective and symptomatological action of memantine relevant for Alzheimer's disease - a unified glutamatergic hypothesis on the mechanism of action. Neurotox Res 2000;2:85-97.

43 Farrimond LE, Roberts E, McShane R. Memantine and cholinesterase inhibitor combination therapy for Alzheimer's disease: a systematic review. BMJ Open 2012;2:e000917.

44 Parsons CG, Danysz W, Dekundy A et al. Memantine and cholinesterase inhibitors: complementary mechanisms in the treatment of Alzheimer's disease. Neurotox Res 2013;24:358-69.

45 Schneider LS, Dagerman KS, Higgins JPT et al. Lack of evidence for the efficacy of memantine in mild Alzheimer disease. Arch Neurol 2011;68:991-8.

46 National Institutes of Health. Osteoporosis prevention, diagnosis, and therapy. NIH Consens Statement 2000 March 27-29;17:1-36.

47 Burger H, de Laet CE, van Daele PL et al. Risk factors for increased bone loss in an elderly population: the Rotterdam Study. Am J Epidemiol 1998;147:871-9.

48 Schuit SC, van der Klift M, Weel AE et al. Fracture incidence and association with bone mineral density in elderly men and women: the Rotterdam Study. Bone 2004;34:195-202.

49 Kanis JA, Johnell O, Oden A et al. Ten year probabilities of osteoporotic fractures according to BMD and diagnostic thresholds. Osteoporos Int 2001;12:989-95.

50 Curtis JR, Safford MM. Management of osteoporosis among the elderly with other chronic medical conditions. Drugs Aging 2012;29:549-64.

51 Reid IR, Bolland MJ, Grey A. Effects of vitamin D supplements on bone mineral density: a systematic review and meta-analysis. Lancet 2014;383:146-55.

52 Wells GA, Cranney A, Peterson J et al. Alendronate for the primary and secondary prevention of osteoporotic fractures in postmenopausal women. Cochrane Database Syst Rev 2008;1;CD001155.

53 Hochberg MC, Thompson DE, Black DM et al. Effect of alendronate on the age-specific incidence of symptomatic osteoporotic fractures. J Bone Miner Res 2005;20:971-6.

54 Yeaw J, Benner JS, Walt JG et al. Comparing adherence and persistence across 6 chronic medication classes. J Manag Care Pharm 2009; 15:728-40.

55 Khosla S, Burr D, Cauley J et al. Bisphosphonate-associated osteonecrosis of the jaw: report of a task force of the American Society for Bone and Mineral Research. J Bone Miner Res 2007;22:1479-91.

56 Meier RP, Perneger TV, Stern R et al. Increasing occurrence of atypical femoral fractures associated with bisphosphonate use. Arch Intern Med 2012;172:930-6.

57 Sellmeyer DE. Atypical fractures as a potential complication of long-term bisphosphonate therapy. JAMA 2010;304:1480-4.

58 Green J, Czanner G, Reeves G et al. Oral bisphosphonates and risk of cancer of oesophagus, stomach, and colorectum: case-control analysis within a UK primary care cohort. BMJ 2010;341:c4444.

59 Cardwell CR, Abnet CC, Cantwell MM et al. Exposure to oral bisphosphonates and risk of esophageal cancer. JAMA 2010;304:657-663.

60 Solomon DH, Patrick A, Brookhart MA. More on reports of esophageal cancer with oral bisphosphonate use. N Engl J Med 2009;360;1789-90.

61 McClung M, Boonen S, Torring $\mathrm{O}$ et al. Effect of denosumab treatment on the risk of fractures in subgroups of women with postmenopausal osteoporosis. J Bone Miner Res 2012;27:211-8. 
62 Papapoulos S, Chapurlat R, Libanati C et al. Five years of denosumab exposure in women with postmenopausal osteoporosis: results from the first two years of the FREEDOM extension. J Bone Miner Res 2012;27:694-701.

63 Cummings SR, San Martin J, McClung MR et al. Denosumab for prevention of fractures in postmenopausal women with osteoporosis. N Engl J Med 2009;361:756-65.

64 Curtis JR, Carbone L, Cheng $\mathrm{H}$ et al. Longitudinal trends in use of bone mass measurement among older Americans, 1999-2005. J Bone Miner Res 2008;23:1061-7.

65 Ettinger B, Black DM, Mitlak BH et al. Reduction of vertebral fracture risk in post- menopausal women with osteoporosis treated with raloxifene: results from a 3-year randomized clinical trial. Multiple Outcomes of Raloxifene Evaluation (MORE) Investigators. JAMA 1999;282:637-45.

66 Chesnut $\mathrm{CH}$ 3rd, Silverman S, Andriano K et al. A randomized trial of nasal spray salmon calcitonin in postmenopausal women with established osteoporosis: the prevent recurrence of osteoporotic fractures study. PROOF Study Group. Am J Med 2000;109:267-76.
67 Traynor K. Experts recommend against calcitonin-salmon for post-menopausal osteoporosis. Am J Health Syst Pharm 2013;70:648-50.

68 Boonen S, Marin F, Mellstrom D et al. Safety and efficacy of teriparatide in elderly women with established osteoporosis: bone anabolic therapy from a geriatric perspective. J Am Geriatr Soc 2006;54:782-9.

69 Cosman F, Lane NE, Bolognese MA et al. Effect of transdermal teriparatide administration on bone mineral density in postmenopausal women. J Clin Endocrinol Metab 2010;95:151-8.

70 Sanders S, Geraci SA. Osteoporosis in postmenopausal women: considerations in prevention and treatment: (women's health series). South Med J 2013;106:698-706.

Address for correspondence: Dr O Mukhtar, King's College Hospital, Denmark Hill, London SE5 9RS, UK.

Email: o.mukhtar@nhs.net 